\title{
Shear-banding predicted by a constitutive model with a structural parameter in cylindrical Couette flows
}

\author{
Mathieu Jenny ${ }^{1,2, \star}$, Sébastien Kiesgen de Richter ${ }^{1,2}$, Nicolas Louvet ${ }^{1,2}$, Naima Gaudel $^{1,2}$, and Salaheddine Skali- \\ Lami $^{1,2}$ \\ ${ }^{1}$ Université de Lorraine, LEMTA, UMR 7563, Vandoeuvre-lès-Nancy, F-54500, France \\ ${ }^{2}$ CNRS, LEMTA, UMR 7563, Vandoeuvre-lès-Nancy, F-54500, France
}

\begin{abstract}
Dense suspensions of non-Brownian particles may partially behave as thixotropic yield stress fluids. We study the flow of such fluids between two concentric cylinders using a phenomenological structural kinetics model. The structural kinetics approach balances the simplicity of phenomenological continuum approaches with a simplified model for structure against the complexity a more fundamental model based on particle micromechanics. A modified version of Houska's model, which includes a diffusive term for the structural parameter, is considered. Depending on the breakdown rate of the structural parameter, shear-banding may be observed. For shear-banding in steady flows, the stress selection depends on the diffusion of the structural parameter. If there is no structural diffusion, the displacement of the interface between the flowing and the static regions fixes the stress at the interface during the transient flow. In the cases of very small diffusive coefficients, the stress at the fluid / solid interface converges to a limit value which is different from the yield stress of the structured material as expected without any diffusion. Nevertheless, the inner torque and the flow profile are quite similar in both cases and the differences are localized near the fluid / solid interface. For shear-banding, the gradients of the structural parameter and the strain rate are very abrupt but the continuity is preserved by the diffusion.
\end{abstract}

\section{Introduction}

Dense suspensions of non-Brownian particles exhibit a very rich behavior such as yield stress and shear-banding $[1,2]$ that remain incompletely understood. Shear-banding has been studied in visco-elastic context $[3,4]$ but recent work [5] exhibits a different mechanism for shear-banding predicted by a visco-plastic thixotropic model. In that context, no-stress jump is associated with shear-banding. The selection of the stress at the flowing-plug interface does not demand to add stress diffusion in the model as it would be expected in visco-elastic context [6]. Fardin et al. [7] show that the length based on this stress diffusion is at the order of magnitude of the molecular size in worm-like micelles. Thus, the diffusion coefficient is expected to be very small $\left(\sim 10^{-10}\right)$. For non-Brownian suspensions, the velocity profiles obtained by Fall et al. [2] suggests that the diffusion length is at the order of magnitude of the particle size, if such diffusive mechanism plays a role in the flow. In our framework, one question arise: does solutions without diffusion term differ from those with a weak diffusion?

It has been shown that the coexistence of static and flowing regions can be associated with the existence of a yield stress which is often related to the existence of a rigid network between the elements of the fluid which has to be

\footnotetext{
^e-mail: mathieu.jenny@univ-lorraine.fr
}

broken for the system starts to flow. Shear banding occurs, in many cases, in systems which exhibit a competition between at least two mechanisms: a breakdown process due to an external applied shear and a spontaneous restructuring of the fluid due to the non-linear interaction between its elements [2, 8]. Ovarlez et al. 2009 [9] and Coussot $\&$ Ovarlez 2010 [10] recently discuss the physical origin of shear localization and shear banding in complex fluids. Authors highlight the existence of a discontinuity of the shear rate profile during shear banding contrary to shear localization where the shear rate goes to zero continuously as one approaches the static region. In the last case, the shear rate is zero on both sides of the static and flowing regions.

The existence of a critical shear rate $\dot{\gamma}_{c}$ in thixotropic yield stress fluids is often explained in terms of an underlying decreasing branch of the flow curve at low shear rates [8]. It is generally admitted that the shear banding may appear in case of a non-monotonic composite curve [3]. Several scenarii can be distinguished: either the shear banding occurs in a flowing material, either it occurs in localized flows between the solid-like and the flowing regions. We focus on the shear banding in localized flows. In such a scenario, the constitutive relation of the material is a decreasing function of the strain rate between 0 and $\dot{\gamma}_{0}$ [see 4 , for instance]. 


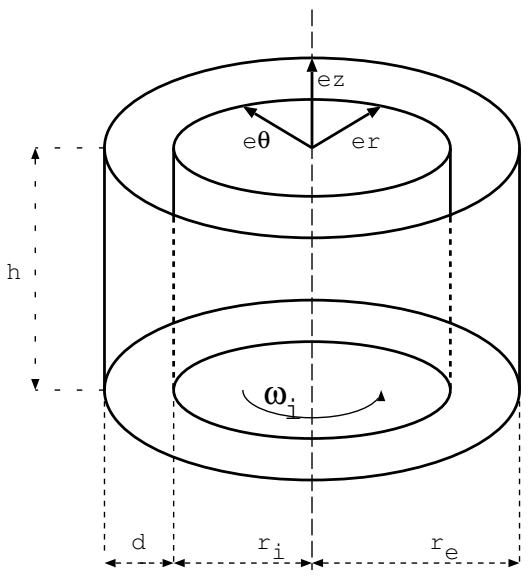

Figure 1. Taylor-Couette geometry. $\mathbf{e}_{r}, \mathbf{e}_{\theta}$ and $\mathbf{e}_{z}$ are the unit vectors of the cylindrical coordinates system $(r, \theta, z)$.

Here, we study shear-banding in cylindrical Couette flows (see geometry on fig. 1) according to the Houska's model [11] modified in order to include a diffusion term. Results obtained with and without structural diffusion are compared, especially when the diffusion coefficient is evanescent.

\section{CONSTITUTIVE EQUATIONS AND NUMERICAL APPROACH}

\subsection{Modified Houska's model}

The Houska's model [11] is built from the HerschelBulkley model, commonly used for non-elastic yield stress fluids, considering that the consistency and the yield stress depend linearly on the structural parameter $\lambda$. To nondimensionalize the constitutive equations of the flow in a cylindrical Couette geometry, we choose the following references: the density of the fluid $\rho$, the velocity of the inner cylinder $v_{i}$ and the gap width $d$. Thus, the structural parameter $\lambda$ is determined by the kinetic equation:

$$
\frac{\partial \lambda}{\partial t}+\mathbf{v} \cdot \boldsymbol{\nabla} \lambda=a^{\star}(1-\lambda)-b^{\star} \lambda \dot{\gamma}^{m}+\mathcal{D}^{\star} \Delta \lambda
$$

where the non-dimensional building and breakdown parameters are

$$
a^{\star}=\frac{a d}{v_{i}} \quad \text { and } \quad b^{\star}=b\left(\frac{v_{i}}{d}\right)^{m-1},
$$

respectively. The non-dimensional structural diffusion coefficient reads:

$$
\mathcal{D}^{\star}=\frac{\mathcal{D}}{v_{i} d} .
$$

The thixotropic breakdown index $m$ is taken equal to 1 in the following. The vector $\mathbf{v}$ denotes the non-dimensional velocity of the fluid.

For the non-Newtonian fluids, several choices may be done for the reference viscosity. In this paper, the reference viscosity is the plastic viscosity of the fluid at the given reference strain rate $v_{i} / d$. The corresponding structural parameter $\lambda_{\text {ref }}$ is given by eq. (1) at equilibrium:

$$
\lambda_{\text {ref }}=\frac{1}{1+b^{\star} / a^{\star}}
$$

Thus, the reference viscosity built using the reference strain rate $v_{i} / d$ reads:

$$
\mu_{\text {ref }}=\mu_{0}\left(1+\Delta K^{\star} \lambda_{\text {ref }}\right)
$$

where $\mu_{0}=K\left(v_{i} / d\right)^{n_{c}-1}$ can be recognized as the standard reference viscosity of a power law fluid with a consistency $K$ and a shear-thinning index $n_{c} . \Delta K^{\star}=\Delta K / K$ is the reduced thixotropic consistency factor.

Using the previous reference dimensions, the NavierStokes and mass conservation equations for incompressible fluids are:

$$
\begin{aligned}
\frac{\partial \mathbf{v}}{\partial t}+(\mathbf{v} \cdot \boldsymbol{\nabla}) \mathbf{v} & =-\boldsymbol{\nabla} p+\frac{1}{R e} \boldsymbol{\nabla} \cdot \overline{\bar{\tau}} \\
\boldsymbol{\nabla} \cdot \mathbf{v} & =0,
\end{aligned}
$$

where $p$ stands for the reduced pressure. The Reynolds number is defined using the reference viscosity (5):

$$
R e=\frac{R e_{0}}{1+\Delta K^{\star} \lambda_{\text {ref }}} \quad \text { where } \quad \operatorname{Re}_{0}=\frac{\rho v_{i} d}{\mu_{0}} .
$$

Thus, the reduced stress tensor reads:

$$
\overline{\bar{\tau}}=\left[\left(\frac{1+\Delta K^{\star} \lambda}{1+\Delta K^{\star} \lambda_{\text {ref }}}\right) \dot{\gamma}^{n_{c}}+B n\left(\frac{1+\tau_{1}^{\star} \lambda}{1+\tau_{1}^{\star} \lambda_{\text {ref }}}\right)\right] \frac{\overline{\bar{\gamma}}}{\dot{\gamma}},
$$

where $\dot{\gamma}$ and $\overline{\overline{\dot{\gamma}}}$ are the non-dimensional strain rate and strain tensor. The equation (9) involves the Bingham number which is the ratio between the yield stress and the plastic viscous stress:

$$
B n=B n_{0} \frac{1+\tau_{1}^{\star} \lambda_{\text {ref }}}{1+\Delta K^{\star} \lambda_{\text {ref }}} \quad \text { where } \quad B n_{0}=\frac{\tau_{0}}{K\left(v_{i} / d\right)^{n_{c}}} .
$$

One can recognize the standard Bingham number $B n_{0}$ of a Herschel-Bulkley fluid with a yield stress $\tau_{y}=\tau_{0}$. The last parameter, $\tau_{1}^{\star}=\tau_{1} / \tau_{0}$, is the reduced thixotropic yield stress.

\subsection{Boundary conditions for the flow}

The inner and outer radii of the Couette setup are defined by $r_{i}=\frac{\eta}{1-\eta}$ and $r_{e}=\frac{1}{1-\eta}$ with $\eta=r_{i} / r_{e}$ the radii ratio. We only consider the case where the inner cylinder rotates and the outer cylinder is fixed, $i . \quad e . \quad \mathbf{v}=v(r) \mathbf{e}_{\theta}$ in the cylindrical basis. Thus, the boundary conditions are:

- At the inner radius, $v\left(r_{i}\right)=1$.

- At the outer radius of the flowing zone, $v\left(r_{o}\right)=0$.

- In our case, there is a material limit at $r=r_{e}$. Thus, the outer radius $r_{o}$ is given by the following criterion: If $\tau\left(r_{e}\right) \geq \tau_{y}, r_{o}=r_{e}$, else, $\tau\left(r_{o}\right)=\tau_{y}$.

- If $\mathcal{D}^{\star} \neq 0$, we have to add the condition of no-diffusion through the surface of the cylinders, $i$. $e . \partial \lambda / \partial r=0$ at $r=r_{i}$ and $r=r_{e}$. 


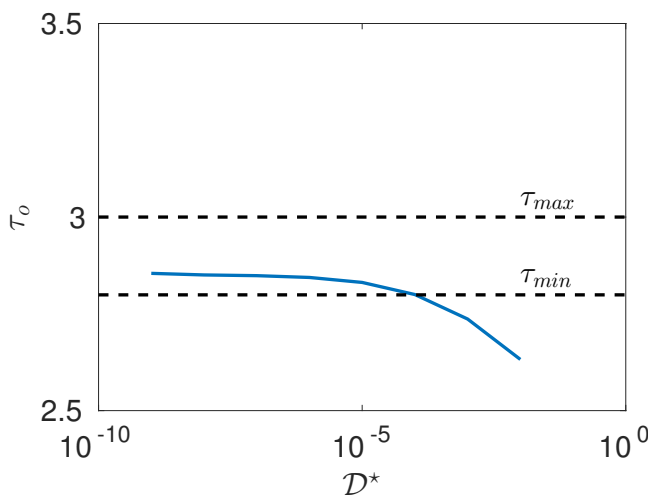

Figure 2. Stress $\tau_{o}$ at the interface for $b^{\star}=2$ (shear-banding) compared to the minimum value of the composite curve $\tau_{\min }$ and the yield stress of the fully structured material $\tau_{\max } v s$ the diffusion coefficient $\mathcal{D}^{\star} . B n=2, n_{c}=1, \Delta K^{\star}=1, \tau_{1}^{\star}=1, a^{\star}=1$.

\subsubsection{Numerical method}

To perform the numerical solution, we use a finite difference method for the spatial discretization. For the derivative operations, the standard second order centered scheme is used. The numerical method used for the spatial discretization is quite well established and is similar to the ones used, for instance, in [5, 12-14]. In order to solve the transient flow, we use an Arbitrary Lagrangian-Eulerian (ALE) method $[15,16]$ for the interface tracking in the partially flowing cases.

\section{Stress selection in shear-banding}

\subsection{Diffusive stress selection}

In the following, we choose the parameters in order to have a localized and shear-banded flow in the gap, i. e. $B n=2$ and $b^{\star}=2$. Considering the stress value at the fluid/solid interface (figure 2), we remark that the stress tends toward a limit value for very small coefficients $\mathcal{D}^{\star}<10^{-4}$ or $10^{-5}$ depending on the breakdown parameter $b^{\star}$. In our case, beyond $b^{\star}=5$, the weakness of the structure dominates and the stress ends to decrease at $r=r_{o}$ when $b^{\star}$ increases.

Nevertheless, for very small diffusive coefficients $\mathcal{D}^{\star}$, the limit value of the selected stress $\tau_{o}$ at $r=r_{o}$ is between the yield stress of the structured material and the minimum value reached by the composite curve (figs. 2 and 3). The local stress (full line in fig. 3) is a monotonic growing function of $\dot{\gamma}$ but the variation of the stress in the range of strain rate between 0 and the apparent strain rate $\dot{\gamma}_{a}$ at the fluid-solid interface is almost flat. Thus, this value would be seen as an apparent yield stress, different from the one observed without shear-banding. In fact, the strain rate falls from the apparent strain rate $\dot{\gamma}_{a}$ down to zero over a distance which is in the order of magnitude of $10 l_{\mathcal{D}}, l_{\mathcal{D}}=\sqrt{\mathcal{D}^{\star} / a^{\star}}$ denoting the diffusion length. This length caracterizes the thickness of the interfacial region where the diffusion term is not negligible (fig. 4).

The diffusion ends to smooth the solution for moderate values of $\mathcal{D}^{\star}$ but, for very small values of $\mathcal{D}^{\star}$, the

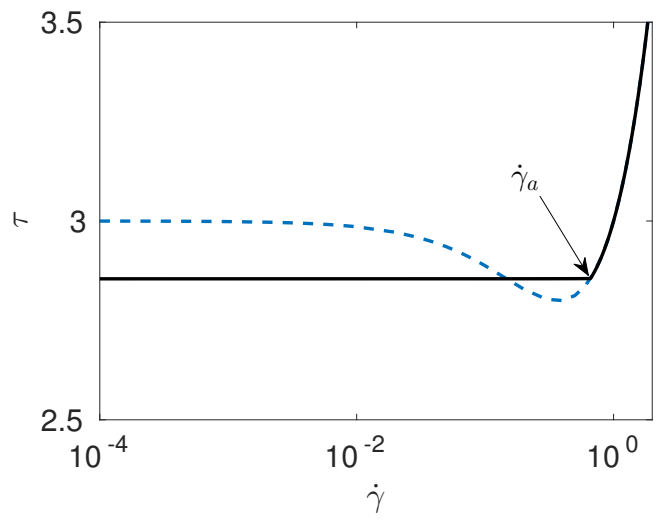

Figure 3. Composite curve (blue dashed line) and local stress in the fluid region (dark full line) $v$ s the strain rate $\dot{\gamma}$ with $\mathcal{D}^{\star}=10^{-9}$ (fig. b). $B n=2, n_{c}=1, \Delta K^{\star}=1, \tau_{1}^{\star}=1, a^{\star}=1, b^{\star}=2$.

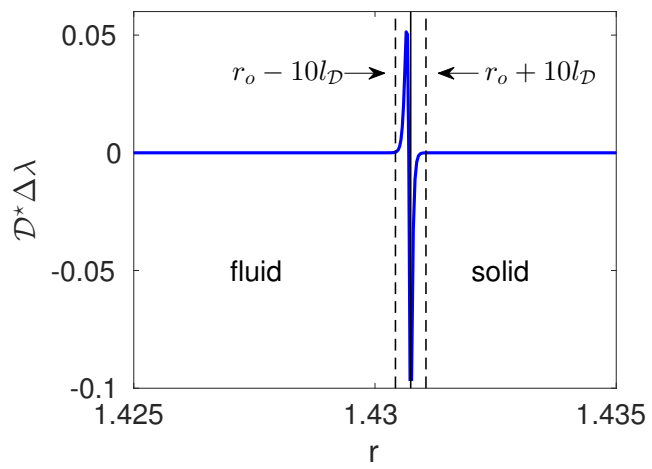

Figure 4. Values of the diffusion term in the gap with $B n=2$, $n_{c}=1, \Delta K^{\star}=1, \tau_{1}^{\star}=1, a^{\star}=1, b^{\star}=2$ and $\mathcal{D}^{\star}=10^{-9} \cdot l_{\mathcal{D}}$ denotes the diffusion length.

sharpness of the field is indistinguishable from the case $\mathcal{D}^{\star}=0$. Moreover, the velocity profile obtained, with only 50 nodes, without any diffusion by setting the value of the selected stress at the interface is indistinguishable from the one provided by the diffusive model (fig. 5). In comparison, up to 16000 nodes are needed both in the flowing and the static regions to catch the diffusion length scale around the interface for $\mathcal{D}^{\star}=10^{-9}$. The non-diffusive model still provides a solution close to the diffusive model for small diffusion coefficients (fig. 5): the interface position $r_{o}$ is only $2.1 \%$ closer to the inner cylinder.

\subsection{Transient flows}

During the transient flow, the inertial term depending on the velocity of the interface insures the continuity of the solution across the flowing / solid-like interface. Thus the stress is well defined at $r=r_{o}$. A brutal cracking of the solid phase of the material at the start of the rotation would allow other stress values than the yield stress but such scenario is beyond the frame of the model considered here. Thus, when $\mathcal{D}^{\star}=0$, the steady equations admit a physical solution where the stress at the interface is the yield stress of the fully structured material even in shear-banded 


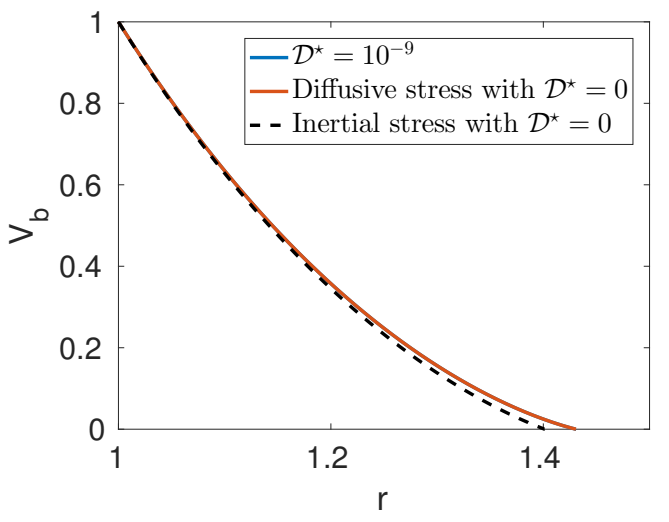

Figure 5. Velocity profile of the steady flow with $B n=2, n_{c}=1$, $\Delta K^{\star}=1, \tau_{1}^{\star}=1, a^{\star}=1, b^{\star}=2$ for a large gap $\eta=0.5$. The computations use 16000 nodes for $\mathcal{D}^{\star}=10^{-9}$ and only 50 nodes for the calculation without diffusion term where the stress is arbitrary set at the interface.

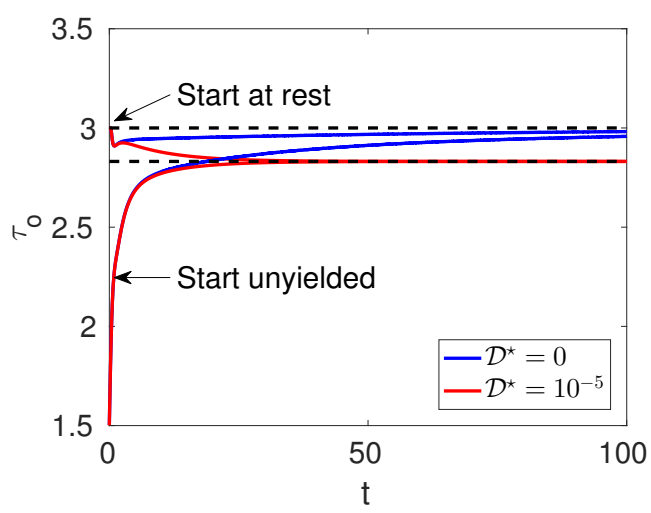

Figure 6. Stress $\tau_{o}$ at the interface $\left(r=r_{o}\right)$. The dashed lines represent the steady limit for $\mathcal{D}^{\star}=0$ and $\mathcal{D}^{\star}=10^{-5}$ respectivelly. Transient flows with $R e=10, B n=2, n_{c}=1, \Delta K^{\star}=1$, $\tau_{1}^{\star}=1, a^{\star}=1$ and $b^{\star}=2$ for a large gap $\eta=0.5$.

flows [5]. Other solutions would imply that the stress at the interface is different from the yield stress.

If $\mathcal{D}^{\star} \neq 0, \partial \tau_{o} / \partial t<0$ before reaching the steady state in the 'start at rest' protocol and the characteristic time of the transient flow is smaller than for $\mathcal{D}^{\star}=0$, even for very small $\mathcal{D}^{\star}$ (fig. 6).

\section{Conclusion}

The diffusive term of the structural parameter selects the stress in case of shear-banded flows. When the diffusion coefficient $\mathcal{D}^{\star}$ is small, typically less than $10^{-5}$, the selected value of the stress does not vary effectively with $\mathcal{D}^{\star}$ and a limit value is reached. In case $\mathcal{D}^{\star}=0$, there is another mechanism to select the stress at the interface in shear-banded flows, assuming that it is always the yield stress of the material. This is imposed by the inertial part of the transient flow where the motion of the interface smooths the transition from the fluidized region to the static region. One can notice that other assumptions for the stress value are beyond the model we consider here. The case $\mathcal{D}^{\star}=0$ may have a meaning for non-Brownian and non-colloidal suspensions although that it is commonly admitted that for colloidal suspensions $\mathcal{D}^{\star} \neq 0$. Prediction of shear-banding and the influence of the structural diffusion are of practical interest to determine the yield stress.

In case of shear-banding, we show that the flows with and without the structural diffusion are different but the solutions remain close to each other. The best way to determine if $\mathcal{D}^{\star} \neq 0$ is to track the shear stress at the interface in shear-banding cases using a planar geometry, used by $\mathrm{S}$. Fielding for instance, where the stress is homogeneous in the gap.

\section{References}

[1] G. Ovarlez, F. Bertrand, S. Rodts, Journal of Rheology 50, 259 (2006)

[2] A. Fall, F. Bertrand, G. Ovarlez, D. Bonn, Phys. Rev. Lett. 103, 178301 (2009)

[3] S.M. Fielding, arXiv preprint arXiv:1512.04715 (2015)

[4] T. Divoux, M.A. Fardin, S. Manneville, S. Lerouge, arXiv preprint arXiv:1503.04130 (2015)

[5] M. Jenny, S. Kiesgen de Richter, N. Louvet, S. SkaliLami, Y. Dossmann, Phys. Rev. Fluids 2, 023302 (2017)

[6] P. Olmsted, O. Radulescu, C.Y. Lu, Journal of Rheology (1978-present) 44, 257 (2000)

[7] M.A. Fardin, O. Radulescu, A. Morozov, O. Cardoso, J. Browaeys, S. Lerouge, Journal of Rheology (1978-present) 59, 1335 (2015)

[8] P.D. Olmsted, Rheologica Acta 47, 283 (2008)

[9] G. Ovarlez, S. Rodts, X. Chateau, P. Coussot, Rheologica Acta 48, 831 (2009)

[10] P. Coussot, G. Ovarlez, The European Physical Journal E 33, 183 (2010)

[11] J. Šesták, R. Žitný, M. Houška, Journal of Food Engineering 2, 35 (1983)

[12] M. Jenny, B. Nsom, Phys. Fluids 19, 108104 (2007)

[13] A.M. Philippe, C. Baravian, M. Jenny, F. Meneau, L.J. Michot, Phys. Rev. Lett. 108, 254501 (2012)

[14] M. Pourjafar, E. Chaparian, K. Sadeghy, Meccanica 50, 1451 (2015)

[15] C. Hirt, A. Amsden, J. Cook, Journal of Computational Physics 14, 227 (1974)

[16] J. Donea, S. Giuliani, J. Halleux, Computer Methods in Applied Mechanics and Engineering 33, 689 (1982) 